\title{
The Calculation of Arias Intensity in North - South Seismic Belt Based on Probabilistic Seismic Hazard Analysis
}

\author{
Weijin $\mathrm{Xu}^{1,2}$, Xuejing $\mathrm{Li}^{2}$, Mengtan $\mathrm{Gao}^{2}$ \\ ${ }^{I}$ China Reinsurance Group Company LTD \\ Beijing, China \\ ${ }^{2}$ Institute of Geophysics, China Earthquake Administration \\ Beijing, China \\ wjxuwin@163.com,1xj-29@163.com,gaomt1957@163.com
}

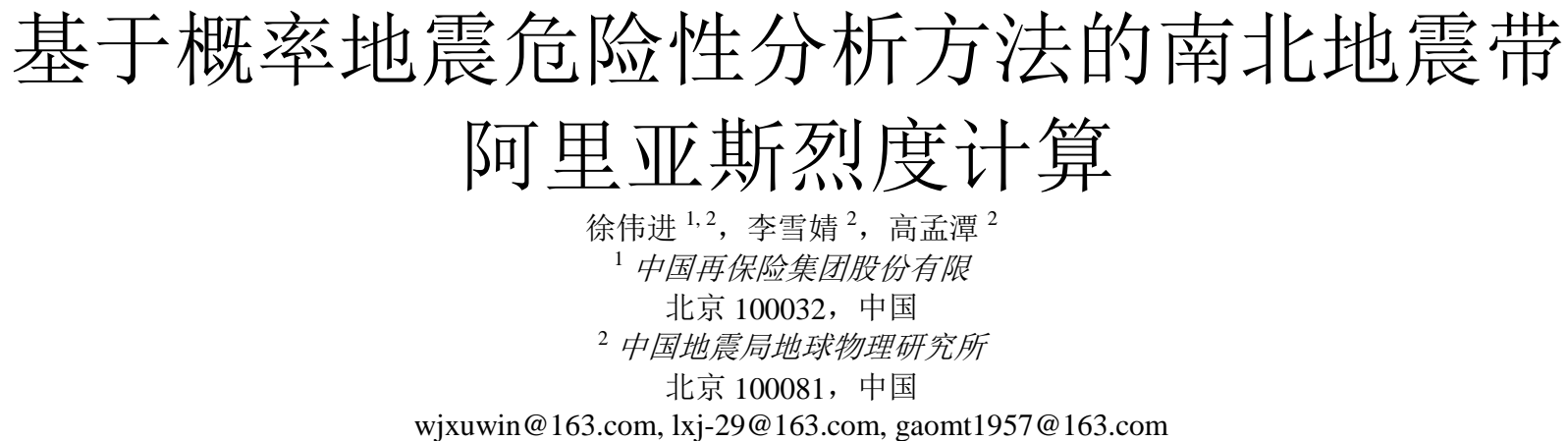

\begin{abstract}
Arias intensity (IA) is an important seismic parameter which contains the information of amplitude, frequencies and duration of ground motion, and which plays an important role in characterizing seismic hazard such as earthquake-induced landslide. In this paper, we carry out the probabilistic seismic hazard analysis based on arias intensity in the North-South seismic belt. We adopt the source areas and seismicity parameters which were used in the fifth generation of the ground motion parameter zoning map of China, and two prediction equations of arias intensity to calculate the values of arias intensity in North-South seismic belt for $10 \%$ probability of exceedance in 50a. The results show that the magnitude of arias intensity is greater than $0.11 \mathrm{~m} / \mathrm{s}$ in most regions of the North-South seismic belt. The provincial capital city and most prefecture-level cities in the seismic zone are located in the region with the value of arias intensity greater than $0.32 \mathrm{~m} / \mathrm{s}$. The values of arias intensity are above $0.54 \mathrm{~m} / \mathrm{s}$ in the region around the main fault zone. This means that the North-South seismic belt have extremely high seismic hazard in earthquakeinduced landslide and liquefaction. It should be strengthened that the evaluation and prevention of earthquake-induced landslide in this area. The results of this paper are of great scientific significance for understanding the seismic hazard of the North-South seismic belt on the other hand.
\end{abstract}

Keywords-Arias Intensity, Seismic Hazard Analysis, Earthquake Induced Landslides, North-South Seismic Belt

摘要一阿里亚斯烈度 (Arias Intensity (IA)) 是一种包 含了地震动振幅、频率以及持时的地震动参数, 在表征地震 诱发滑坡等地震灾害危险性方面具有重要的指示意义。本文 在南北地震带开展了基于阿里亚斯烈度的概率地震危险性分 析。文中采用了编制第五代中国地震动参数区划图的地震活 动性模型, 引入阿里亚斯烈度预测方程, 基于概率地震危险 性分析方法计算了南北地震带 50 年超越概率 $10 \%$ 下的阿里亚 斯烈度。研究结果表明, 南北地震带绝大部分区域的阿里亚 斯烈度值大于 $0.11 \mathrm{~m} / \mathrm{s}$; 地震带内的省会城市和大部分地级市
位于其阿里亚斯烈度值大于 $0.32 \mathrm{~m} / \mathrm{s}$ 的区域; 主要断裂带周 围的阿里亚斯烈度值在 $0.54 \mathrm{~m} / \mathrm{s}$ 以上。这意味着南北地震带 具有极高的地震诱发滑坡和砂土液化的危险性, 应当加强对 该地区地震诱发滑坡的评估和防范。本文的研究结果对于从 更多方面认识南北地震带的地震危险性、对地震灾害的预测 预防以及地震保险方案的制定具有重要的科学意义和应用价 值。

关键词：阿里亚斯烈度, 地震危险性分析, 地震诱发滑 坡, 南北地震带

\section{I. 引言}

阿里亚斯烈度（Arias Intensity (IA)）是一种包含了 地震动振幅、频率成分以及持时的地震动参数, 其对某 些地震破坏的预测可能比仅依靠地震动的振幅更具可靠 性[1], 可以有效地捕获潜在地震的破坏。阿利亚斯烈度 能够反映宽频带上地震信号的能量特征, 即地震动整个 持时的影响 [2-3]。阿里亚斯烈度的这一特征使其可以应 用到地震对结构或岩土工程的破坏预测中去, 已有研究 表明其与受地震动高频影响的结构地震反应、砂土液化 以及地震诱发滑坡等具有相关性。如 2003 年,

Travasarou 等[2]证明了采用阿里亚斯烈度对短周期建筑 结构进行可能的破坏预测是有效的, 研究还表明阿里亚 斯烈度与结构破坏的相关性比峰值加速度与结构破坏的 相关性要强。更重要的, 科学家们的研究还表明阿里亚 斯烈度与地震诱发滑坡具有很强的相关性 [4-6], 利用这 一特征可以对潜在的地震诱发滑坡进行预测。也可以用 来估计地震导致场址砂土液化的可能性[7], 从而对地震 导致的地基失效进行预测 [8]。

由上述介绍可知开展基于阿里亚斯烈度的概率地震 危险性分析具有十分重要的科学意义和应用价值。目前 科学家们已经建立了数个阿里亚斯烈度预测方程 1-3, 9- 
10], 可以应用到地震危险性分析中。国际上已有许多学 者开展了基于阿里亚斯烈度的概率地震危险性分析 [1114]。本研究我们将在我国的南北地震带开展基于阿里亚 斯烈度的概率地震危险性分析。南北地震带是中国大陆 中部一条贯穿南北的地震密集带, 他也是一条由一系列 近 SN 向断裂组成的结构十分复杂的地质构造带[15]。南 北地震带(构造带)北起阴山和河套盆地, 向南经过银川盆 地、六盘山、西秦岭、龙门山松潘甘孜、西康地区, 直 到滇西三江, 这里地势险要、人口比较密集, 但是地震 等各种地质灾害频发 [16]。南北地震带地震活动非常频 繁, 发生了中国大陆有历史记录以来近 $40 \%$ 以上的 7 级 以上地震和近半数的 8 级以上地震 [17], 现代仪器记录 的小震也非常密集, 地震危险性水平很高。南北地震带 也是我国西部经济发展水平高, 大中城市集中, 人口稠密 地区, 更是我国水利电力资源, 国防尖端工程等非常集中 的重要地区, 一旦发生严重破坏性地震, 将会产生重大灾 害[18]。如 2008 年汶川地震和 2013 年芦山地震导致的 山体滑坡均造成了严重的人员伤亡和财产损失[19-21]。 特别是 2008 年汶川地震导致的山体滑坡造成了极为严重 的人员伤亡 [22], 震区多地产生的砂土液化也对建筑物 造成了较为严重的破坏[23]。因此, 在南北地震带开展 基于阿里亚斯烈度的概率地震危险性分析, 对于评估南 北地震带地震诱发滑坡和沙土液化危险性以, 及从另一 个角度认识该地区的地震危险性具有重要作用。

\section{II. 阿里亚斯烈度及其衰减模型}

从数学上来说, 阿里亚斯烈度是将加速度的平方对 整个时程进行积分。在物理上, 描述的是频率均匀分布 的一组简谐振子每单位质量所储存的总能量, 其在强地 面运动中 $\mathrm{x}$ 方向的表达式可写成[24]:

$$
I_{a}=\frac{\pi}{2 g} \int_{0}^{\infty} a_{x}(t)^{2} d t
$$

其中 $a_{x}(t)$ 是 $x$ 方向的加速度时程, 单位取 $\mathrm{m} / \mathrm{s}^{2}, \mathrm{~g}$ 为重力加速度 $\left(\mathrm{m} / \mathrm{s}^{2}\right)$ 。根据定义我们可知, 阿里亚斯烈度 是一个二阶张量, 其矩阵的迹是一个不变量, 即在通过 某一原点的任一直角坐标系下均具有相同的阿里亚斯烈 度值。由于坚向地震动的阿里亚斯烈度值相对较小, 且 地震工程学中人们更加关心水平向的地震动, 因此一般 情况下, 人们是分别计算水平向相互垂直的强震记录的 阿里亚斯烈度值, 并计算二者的算术平均值, 根据定义 可知, 二者之和是一个不依赖于坐标轴选取的定值。

(1) 式中的 $I_{a}$ 是指:

$$
I_{a}=\frac{I_{x x}+I_{y y}}{2}
$$

其中 $I_{x x}, I_{y y}$ 是根据强震记录中两个相互垂直的水平 向分量计算得到的。

目前使用最为广泛的阿里亚斯烈度衰减模型是 Travasarou 等 [2]建立的, 其表达式为:

$$
\begin{aligned}
\ln \left[I_{a}\right]= & c_{1}+c_{2}(M-6)+c_{3} \ln (M / 6)+c_{4} \ln \left(\sqrt{R^{2}+h^{2}}\right)+\left(s_{11}+s_{12}(M-6)\right) S_{C} \\
& +\left(s_{21}+s_{22}(M-6)\right) S_{D}+f_{1} F_{N}+f_{2} F_{R}
\end{aligned}
$$

其中 $c, h, s, f$ 均为回归参数, 可根据强震数据进行 估算。 $M$ 为震级, $R$ 为距离。 $S_{C}, S_{D}$ 表示场地类型的参 数, 当为 $\mathrm{C}$ 类场地时 $S_{C}=1, S_{D}=0$, 当为 $\mathrm{D}$ 类场地时 $S_{C}=0, S_{D}=1$, 基岩场地时 $S_{C}=0, S_{D}=0 。 F_{N}, F_{R}$ 为表示 震源机制的参数, 当正断层时 $F_{N}=1, F_{R}=0$, 当逆断层时 $F_{N}=0, F_{R}=1$, 走滑断层时 $F_{N}=0, F_{R}=0$ 。本研究中我们 采用 Travasarou et al.[2]的 Ia 衰减模型计算了整个南北地 震带的阿里亚斯烈度危险性。

\section{III. 地震潜在震源区及活动性参数}

潜在震源区和地震活动性参数是概率地震危险性计 算的基本输入。潜在震源区是指那些具有潜在的发生破 坏性地震的区域。在中国, 潜在震源区采用三级划分原 则, 即先划分大的地震区 (带), 在地震带内再划分较 小的地震构造区, 最后再划分潜在震源区。潜在震源主 要是依据地质、地震活动性、大地测量以及地球物理场 等资料来进行区划分的。在中国, 潜在震源区是未来发 生地震宏观震中的集合。地震活动性参数主要有地震发 生率、 $b$ 值、震级上限级震源深度等, 主要是根据地震 活动性、地质以及断层滑动速率等资料进行估算的。本 研究中采用的潜在震源区是编制《中国地震动参数区划 图》所构建的潜在震源区 (图 1)。地震带及潜在震源 区的活动性参数可从 《中国地震动参数区划图宣贯教 材》[25]中查阅获取。

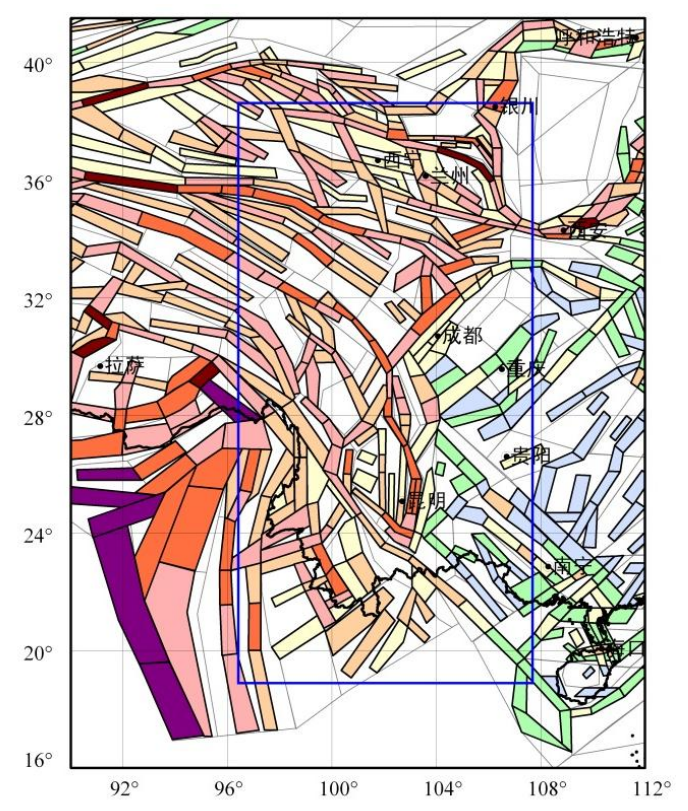

图 1 南北地震带及其周缘地区潜在震源区模型

\section{IV. 概率地震危险性计算方法及流程}

在概率地震危险性分析中, 某一场点的地震危险性 是对所有可能对该场点产生破坏的潜在地震的危险性进 行积分, 即: 


$$
\begin{aligned}
& v\left(u>u_{0}\right)=\sum_{i=1}^{N_{\text {source }}} N_{i}\left(M>M_{\min }\right) \\
& \int_{M_{\min }}^{M_{\max }} \int_{r=0}^{\infty} f_{m i}(M) f_{r i}(r, M) P\left(u>u_{0} \mid M, r\right) d r d M
\end{aligned}
$$

其中 $v\left(u>u_{0}\right)$ 为地震危险性的超越概率, 即潜在破坏 地震产生的地震动 $u$ 大于给定目标地震动 $u_{0}$ 的概率。本 文中 $u$ 和 $u_{0}$ 均为阿里亚斯烈度, $u$ 为根据地震动预测方 程计算的阿里亚斯烈度值, $u_{0}$ 为给定的一系列目标阿里 亚斯烈度值。在实际计算中, 人们一般认为 $M>M_{\text {min }}$ 的 地震对场点有破坏, $M<M_{\min }$ 的地震对场点没有破坏, 一般情况下 $M_{\text {min }}$ 取 4.0 。 $N_{\text {source }}$ 为潜在震源区的数量, $N_{i}$ 为第 $i$ 个潜在震源区震级大于 $M_{\text {min }}$ 地震的发生率。 $f(r)$ 为 距离的概率密度函数, 在实际计算中, 对距离的积分可 表示为对所有距离上的对场点危险性有贡献的潜在震源 进行累加。 $f(M)$ 为震级的概率密度函数, 其是根据震级频度关系式 $\lg N_{I}(M)=a-b M$ [26]推导而来, 可写为:

$$
f(M)=\frac{b \ln 10 \cdot 10^{-b\left(M=M_{\min }\right)}}{1-10^{-b\left(M_{u}-M_{\min }\right)}}
$$

式中 $P\left(u>u_{0} \mid M, r\right)$ 为在距离 $r$ 处震级为 $M$ 的地震产生 的阿里亚斯烈度值 $u$ 大于给定目标值 $u_{0}$ 的概率。

\section{V. 结果分析}

根据上文介绍的地震潜在震源区、阿里亚斯烈度预 测模型以及概率地震危险性计算方法计算了南北地震带 50 年超越概率 $10 \%$ 概率水平下（复发周期 475 年）的阿 里亚斯烈度值，并给出了空间分布等值线图（图 2）。 我们在等值线图中特别标出了 $0.11 \mathrm{~m} / \mathrm{s} 、 0.32 \mathrm{~m} / \mathrm{s}$ 和 $0.54 \mathrm{~m} / \mathrm{s}$ 三个数值档。根据前人的实验研究, 这三个阿里 亚斯烈度值分别对应着三种类型的地震诱发滑坡 [4]:

（1）当 Ia 值大于 $0.11 \mathrm{~m} / \mathrm{s}$ 时, 斜坡上的滑块会出现纵向 滑动掉落, 最常见的是碎片的崩落和雪崩。(2) 当 Ia 值大于 $0.32 \mathrm{~m} / \mathrm{s}$ 时, 斜坡上的滑块会剧烈滑动掉落, 表 现为石块、土等会快速纵向滑动, 产生泥石流。（3）

当 Ia 值大于 $0.54 \mathrm{~m} / \mathrm{s}$ 时, 滑块的运动会向横向扩展并快 速剧烈滑动, 表现为产生大规模山体滑坡。上述三个阈 值已被学界广泛接受用于评估地震诱发滑坡可能性的大 小[12]。图 2 是根据 Travasarou 和 Bray[2]的阿里亚斯列 预测方程计算的南北地震带 Ia 分布, 从图上我们可以看 出南北地震带绝大部分地区 Ia 值都在 $0.11 \mathrm{~m} / \mathrm{s}$ 以上, 在 主要断裂带周缘 Ia 值都在 $0.54 \mathrm{~m} / \mathrm{s}$ 以上, 具有极高的地 震诱发滑坡风险。对于研究区内省会城市以及大部分的 地级市 Ia 值大于 $0.32 \mathrm{~m} / \mathrm{s}$ 的区域, 应当加强该区域地震 诱发滑坡的评估及防范。

本文中计算的南北地震带阿里亚斯烈度值与《中国地 震动参数区划图》的加速度值在空间分布上具有很强的 相关性。一方面是由于二者采用的潜在震源区是相同 的。另一方面, 根据对强震记录的研究表明, 峰值加速 度（PGA）与阿里亚斯烈度具有很强的正相关性[23]。
VI. 结论与讨论

本文采用编制《中国地震动参数区划图》的潜在震 源区及地震活动性参数, 基于 Travasarou 和 Bray[2]的阿 里亚斯烈度预测模型, 计算了南北地震带的 50 年超越概 率 $10 \%$ 下的 Ia 分布, 得出以下结论: 南北地震带绝大部 分地区 Ia 值高于 $0.11 \mathrm{~m} / \mathrm{s}$; 大多数省会城市和地级市位 于 Ia 大于 $0.32 \mathrm{~m} / \mathrm{s}$ 的区域; 在高震级上限潜在震源周围 Ia 值大于 $0.54 \mathrm{~m} / \mathrm{s}$ 以上。由此可见, 该区域具有极高的 地震诱发滑坡风险, 因此应当对南北地震带、特别是 Ia 值高的城镇地区展开地震诱发滑坡评估, 做好地震诱发 滑坡的防范工作。

本文的研究结果扩展了我们对南北地震带地震危险 性的认识, 对于我们进一步认识南北地震带的地震危险 性具有重要的科学意义。

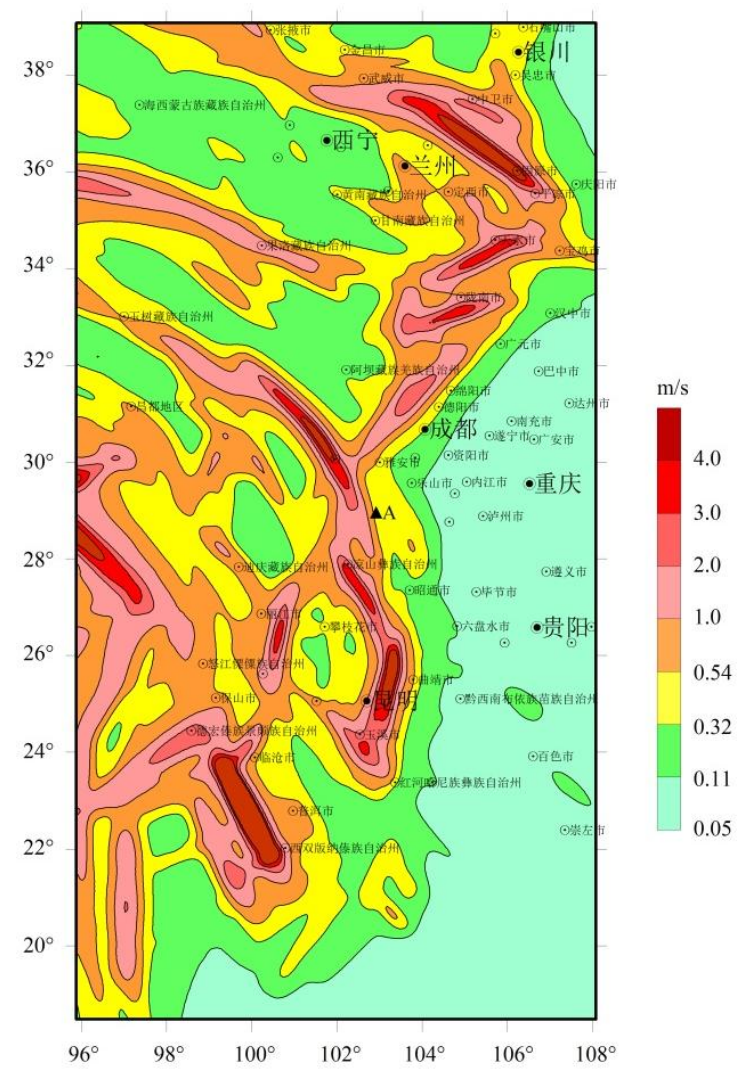

图 2 南北地震带 50 年超越概率 $10 \%$ 下阿里亚斯烈度分 布, 根据 Travasarou and Bray[2]模型计算

致谢

本研究受科技部重点研发计划项目《地震保险损失 评估模型及应用研究》（编号 2018YFC1504600) 资 助。

\section{参考文献}

[1] Piggott, R. F., and P. J. Stafford, "A predictive model for Arias intensity at multiple sites and consideration of spatial correlations, Earthq. Eng. Struct. Dynam,” vol 41, issue 3, pp. 431-451, 2012.

[2] Travasarou, T., and J. D. Bray, "Empirical attenuation relationship for Arias intensity," Earthq. Eng. Struct. Dynam, vol 32, pp. 1133-1155, 2003. 
[3] Stafford, P. J., J. B. Berrill, and J. R. Pettinga, "New predictive equations for Arias intensity from crustal earthquakes in New Zealand,"J. seismol, vol 13, pp.31-52, 2009.

[4] Harp EL, Wilson RC., "Shaking intensity thresholds for rock falls and slides: evidence from 1987 Whittier Narrows and Superstition Hills earthquake strong - motion records," Bulletin of the Seismological Society of America, vol 85, pp.1739-1757, 1995.

[5] Keefer DK., "Investigating landslides caused by earthquakes - a historical review," Surveys in Geophysics, vol 23, pp.473-510,2002.

[6] Jibson RW., "Regression models for estimating coseismic landslide displacement," Engineering Geology, vol 91, pp.209-218, 2007.

[7] Egan JA, Rosidi D., "Assessment of earthquake - induced liquefaction using ground - motion energy characteristics," Proceedings of the Pacific Conference on Earthquake Engineering, Auckland, New Zealand, 1991.

[8] Kramer SL. "Geotechnical Earthquake Engineering," Prentice Hall: Upper Saddle River, NJ, 1996.

[9] Lee, C. T., B. S. Hsieh, C. H. Sung, and P. S. Lin, "Regional Arias intensity attenuation relationship for Taiwan considering VS30," Bull. Seismol. Soc. Am. vol 102, pp.129-142, 2012.

[10] Campbell, K.W., and Y. Bozorgnia, "A comparison of ground motion prediction equations for Arias intensity and cumulative absolute velocity developed using a consistent database and functional form, Earthq," Spectra , vol 28, issue 3, pp.931-941,2012,

[11] Abdrakhmatov K., H.-B. Havenith, D. Delvaux, D. Jongmans \& P. Trefois, "Probabilistic PGA and Arias Intensity maps of Kyrgyzstan (Central Asia)," Journal of Seismology, vol 7, pp.203-220, 2003.

[12] Peláez J. A., José Delgadob, Carlos López Casado, “A preliminary probabilistic seismic hazard assessment in terms of Arias intensity in southeastern Spain,” Engineering Geology, vol 77, pp.139-151, 2005.

[13] Howard J.K., P.G., E.G., W.A. Fraser, P.G., E.G., and M.G. Schultz P.E, "Probabilistic Use of Arias Intensity in Geotechnical Earthquake
Engineering," Geotechnical Earthquake and Engineering and Soil Dynamics IV Congress 2008. GSP 181, 2008 ASCE

[14] Ghodrati Amiri G., H. Mahmoodi, and S. A. Razavian Amrei, "Seismic Hazard Assessment of Tehran Based on Arias Intensity," AIP Conference Proceedings vol. 1020, issue 270, 2008.

[15] 王椿镛，杨文采，吴建平等，“南北构造带岩石圈结构与地震的研 究. 地球物理学报,”vol 58, issue 11, pp. 3867-3901, 2005.

[16] 刘光鼎, “加强地震带构造研究, 促进社会可持续发展. 地球物理 学报,”vol 58, issue 11, pp. 3865-3866, 2015.

[17] 汪一鹏, 马瑾, 李传友, “南北地震带强震迁移特征及其与南亚地震 带的关系, ”地震地质, vol 29, issue 1, pp. 1-14, 2007.

[18] 杨建思, 黄文辉, 徐志强等, “南北地震带地震强化监测及技术途径 一动态虚拟数字地震台网技术, ”地震地磁观测与研究, vol 30, issue 5, pp.51-58, 2009

[19] 许强, 李为乐, “汶川地震诱发大型滑坡分布规律研究, ”工程地质 学报, vol 18, issue 6,pp.818-827, 2010 .

[20] 许冲，徐锡伟，郑文俊，等，“2013 年四川省芦山“4. 20”7. 0 级强 烈地震触发滑坡,”地震地质, vol 35, issue 3, pp.641-660, 2013.

[21] 陈晓利, 袁仁茂, 属露, “Newmark 方法在芦山地震诱发滑坡分布预 测研究中的应用,”地震地质, vol 35, issue 3, pp. 661-670,2013.

[22] 李秀珍, 孔纪名, ““5.12”汶川地震诱发典型滑坡的类型和特征,”山 地学报. Vol 29, issue 5, pp.598-607,2011

[23] Liu J M, Wang T, Wu S, Gao MT., "New Empirical Relationships between Arias Intensity and Peak Ground Acceleration," Bulletin of the Seismological Society of America, vol 106, issue 5, pp.1-9, 2016.

[24] Arias, A. , "Measure of earthquake intensity, in Seismic Design for Nuclear Power Plants," R. J. Hansen (Editor), Massachusetts Institute of Technology Press, Cambrige, Massachusetts, pp.438-483,1970.

[25] 高孟潭, “GB18306-2015《中国地震动参数区划图》宣贯教材”. 北 京: 中国标准出版社, 2015.

[26] Gutenberg, B., and C. F. Richter, "Frequency of earthquakes in California," Bull. Seism. Soc. Am., vol 34, pp.185-188, 1944. 